\title{
Utilización de las series de animación como recurso didáctico en la elaboración de casos. Análisis de una experiencia en la innovación docente en la Universitat Politècnica de València
}

\begin{abstract}
Francisca Ramón Fernández ${ }^{a}$, Vicente Cabedo Mallol ${ }^{b}$, María Emilia Casar Furióc, Vicent Giménez Chornet ${ }^{\mathrm{d}}$ y Juan Vicente Oltra Gutiérrez

${ }^{a}$ Profesora titular. Departamento de Urbanismo, Universitat Politècnica de València,

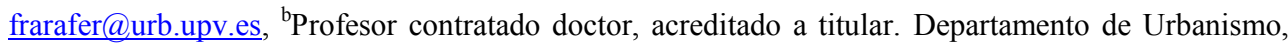
Universitat Politècnica de València, vicamal@urb.upv.es,${ }^{\mathrm{c}}$ Profesora contratada doctora, acreditada a titular. Departamento de Urbanismo, Universitat Politècnica de València, macafu@urb.upv.es, ${ }^{\mathrm{d}}$ Profesor titular. Departamento de Comunicación Audiovisual, Documentación e Historia del Arte, Universitat Politècnica de València, vigicho@har.upv.es, ${ }^{\mathrm{e}}$ Profesor titular de Escuela Universitaria. Departamento de Organización de Empresas, Universitat Politècnica de València, jvoltra@omp.upv.es
\end{abstract}

\begin{abstract}
In the present work there is analyzed the utilization of series of animation for the design of practical cases and the results obtained in the educational area after the concession of a project of educational innovation (PIME) on the part of Universitat Politècnica de València, for the year 2015.

The obtained results demonstrate the acceptance on the part of the pupil and the reflection of juridical questions applied to the reality, as well as that allows us the application of the transverse competition CT-06. Teamwork and leadership, with the exhibitions in the classroom of the different practical elaborated cases.

With it we think that the comprehension of concepts is improved substantially by the utilization of cartoon and description of situations in series as the Simpsons, known by all, and that facilitate the application of the legislation in the raised conflicts.
\end{abstract}

Keywords: cartoon, cases, teaching resource, law, teaching innovation

\section{Resumen}

En el presente trabajo se analiza la utilización de series de animación para el diseño de casos prácticos y los resultados obtenidos en el ámbito docente tras la concesión de un proyecto de innovación docente (PIME) por parte de la Universitat Politècnica de València, para el año 2015.

Los resultados obtenidos demuestran la aceptación por parte del alumno y la reflexión de cuestiones jurídicas aplicadas a la realidad, así como que nos permite la aplicación de la competencia transversal CT-06. Trabajo en equipo y liderazgo, con las exposiciones en el aula de los distintos casos prácticos elaborados. 
Con ello consideramos que se mejora sustancialmente la comprensión de conceptos con la utilización de dibujos animados y descripción de situaciones en series como los Simpsons, conocidas por todos, y que facilitan la aplicación de la legislación en los conflictos planteados.

Palabras clave: series de animación, casos, recurso didáctico, legislación, innovación docente

\section{Introducción}

El presente trabajo tiene como fundamento mostrar los resultados del Proyecto de Innovación y Mejora Educativa (PIMEs) concedido a la propuesta «Utilización de las series de animación como recurso didáctico en la elaboración de casos», y que obtuvo resolución favorable de la Comisión de Evaluación y Seguimientos de Proyectos de Innovación y Convergencia Europa (CESPIC), en el año 2015, participando distintos integrantes del grupo de innovación docente (EICE) RETAJUDOCA (Recursos tecnológicos para el aprendizaje jurídico, la documentación y comunicación audiovisual).

Teniendo en cuenta la importancia del sistema de casos en las asignaturas de carácter jurídico impartidas en titulaciones no jurídicas, se considera oportuno diseñar una serie de actividades tomando como base series de animación. Por ello, se motiva al alumno a pensar sobre una posible solución aplicable, y se utilizan las nuevas tecnologías de la información y la comunicación (TICs) como dinamizadores de unas prácticas tradicionalmente estandarizadas.

Todo ello se basa en la utilización de las nuevas tecnologías para el trabajo del alumno (CANÓS y RAMÓN, 2005), así como la implantación de éstas nuevas tecnologías en el aprendizaje de disciplinas de naturaleza jurídica en carreras técnicas (CANÓS y RAMÓN, 2007), en función también del cambio de roles (CANÓS, RAMÓN y ALBALADEJO, 2008; CANÓS, RAMÓN y ALBALADEJO 2009) y con la finalidad de incrementar la calidad docente (CANÓS y RAMÓN, 2009).

Habitualmente se suelen diseñar casos prácticos en el ámbito jurídico con sentencias o fragmentos legislativos contextualizados en un argumento. No obstante, es un hecho que el visionado de series de animación facilita la comprensión de problemas cotidianos. Un ejemplo de ello es la serie de los Simpsons (MARTÍNEZ, 2004) que se desarrolla en la época actual (LAZO y TOVAR, 2011) y que se contextualiza en una familia y que es seguida de forma habitual por un numeroso público, por su diversión e ironía (ROMERO, 2006 y ANTINORA, 2010).

La innovación propuesta aúna audiovisual y argumentos jurídicos para aportar soluciones a conflictos planteados. La idea de partir del visionado de series de dibujos animados que son conocidas y fácilmente identificables por el público ha sido por considerar esta opción como recursos didáctico para dinamizar la docencia en el aula (ÍÑIGO, 2012).

(cc) EY-NC-ND 2016, Universitat Politècnica de València 
La utilidad de las series de animación también radica en la forma de abordar cada uno de los temas (PORTILLO, 2015), ya que ello nos permite ver cómo enfoca el alumnado la resolución del conflicto planteado.

\section{Objetivos}

El principal objetivo planteado es diseñar un repertorio de casos prácticos teniendo en cuenta la multidisciplinariedad de los profesores participantes, y la diversidad de las asignaturas en las que se van a aplicar, tomando como soporte audiovisual una serie de animación.

La pretensión es que mediante el visionado de un episodio de una serie animada, el alumno pueda resolver un caso práctico en relación con un contenido de una asignatura. Fomenta la reflexión sobre lo acontecido en el audiovisual y su aplicación a la realidad, ya que se le formulan distintas preguntas al alumno para aplicar la legislación correcta y aportar soluciones a los conflictos planteados.

Como objetivos específicos de la utilización de series de animación para el diseño de casos prácticos, destacamos los siguientes:

a) Potenciación de la utilización de recursos audiovisuales en el aula, teniendo en cuenta las TICs como métodos de innovación docente.

b) Contextualización de situaciones reales en series de animación donde se reflejan aspectos sociales contemporáneos.

c) Planteamiento de problemas para aporte de soluciones no únicas a los conflictos planteados.

d) Aplicación de legislación y posicionamiento en una de las orientaciones marcadas por la normativa.

\section{Desarrollo de la innovación: la utilización de series de animación para diseñar casos prácticos}

\subsection{Asignaturas en las que se desarrolla la innovación}

En este punto vamos a ver qué asignaturas se han escogido para la aplicación de la innovación docente, así como el tipo de asignatura, número de créditos y alumnos, y profesorado, departamento y titulación en la que se imparte.

Como se puede observar en la siguiente tabla, todas las asignaturas son de carácter obligatorio y se imparten en grado y máster, con un número considerable de alumnos. Destaca que la naturaleza de las asignaturas son de ámbito legislativo o un matiz jurídico, pero impartidas todas en titulaciones no jurídicas. Ello significa que el perfil del alumno no es de Derecho, por lo que el diseño de casos prácticos le ayuda a la comprensión de problemas de ámbito jurídico que se pueden plantear en la vida real.

(cc) EY-NC-ND 2016, Universitat Politècnica de València

Congreso In-Red (2016) 
Tabla 1. Listado asignaturas involucradas

\begin{tabular}{|c|c|c|c|c|c|c|c|}
\hline Código & Nombre & Carácter & $\begin{array}{l}\text { Núms } \\
\text { ECTS }\end{array}$ & $\begin{array}{l}\text { Nums. } \\
\text { alumnos }\end{array}$ & $\begin{array}{l}\text { Departamento } \\
\text { responsable }\end{array}$ & $\begin{array}{l}\text { Titulación } \\
\text { en que se } \\
\text { imparte }\end{array}$ & Profesor \\
\hline 31247 & $\begin{array}{l}\text { Gestión } \\
\text { empresarial de } \\
\text { la edificación }\end{array}$ & Obligatoria & 2 & 15 & Urbanismo & $\begin{array}{l}\text { Máster en } \\
\text { Edificación }\end{array}$ & $\begin{array}{l}\text { Francisca } \\
\text { Ramón } \\
\text { Fernández }\end{array}$ \\
\hline 32829 & $\begin{array}{l}\text { Gestión del } \\
\text { conocimiento y } \\
\text { recursos } \\
\text { informáticos }\end{array}$ & Obligatorio & 5 & 40 & $\begin{array}{l}\text { Comunicación } \\
\text { Audiovisual, } \\
\text { Documentación } \\
\text { e Historia del } \\
\text { Arte }\end{array}$ & $\begin{array}{l}\text { Máster en } \\
\text { Gestión } \\
\text { Cultural }\end{array}$ & $\begin{array}{l}\text { Vicent } \\
\text { Giménez } \\
\text { Chornet }\end{array}$ \\
\hline 10028 & $\begin{array}{l}\text { Gestión } \\
\text { urbanística }\end{array}$ & Obligatoria & 14.19 & 400 & Urbanismo & $\begin{array}{l}\text { Grado en } \\
\text { Gestión de } \\
\text { la } \\
\text { Edificación }\end{array}$ & $\begin{array}{l}\text { María } \\
\text { Emilia } \\
\text { Casar } \\
\text { Furió }\end{array}$ \\
\hline 34015 & $\begin{array}{l}\text { Marco legal y } \\
\text { deontológico de } \\
\text { la información }\end{array}$ & Obligatoria & 3 & 50 & $\begin{array}{l}\text { Organización } \\
\text { de empresas }\end{array}$ & $\begin{array}{l}\text { Máster en } \\
\text { gestión de } \\
\text { la } \\
\text { información }\end{array}$ & $\begin{array}{l}\text { Juan } \\
\text { Vicente } \\
\text { Oltra } \\
\text { Gutiérrez }\end{array}$ \\
\hline 11550 & $\begin{array}{l}\text { Deontología } \mathrm{y} \\
\text { Profesionalismo }\end{array}$ & Obligatoria & 4.5 & 400 & $\begin{array}{l}\text { Organización } \\
\text { de empresas }\end{array}$ & $\begin{array}{l}\text { Grado en } \\
\text { Informática }\end{array}$ & $\begin{array}{l}\text { Juan } \\
\text { Vicente } \\
\text { Oltra } \\
\text { Gutiérrez }\end{array}$ \\
\hline
\end{tabular}

Fuente: Elaboración propia (2016)

Por tanto, el número de alumnos a los que se les ha podido aplicar la dinámica de los casos prácticos ha sido durante el curso académico 205-2016 ha sido de un total de 473 alumnos, ya que en el resto de asignaturas no ha sido posible su aplicación por distintos motivos de organización de la docencia.

\section{Resultados de la utilización de los casos prácticos: mejoras e interactividad}

\subsection{Los casos prácticos basados en series de animación y la competencia transversal} de trabajo en equipo y liderazgo 
Vamos a ver en este punto cómo nos ha permitido la utilización de los casos prácticos basados en series de animación aplicar la competencia transversal CT-06, trabajo en equipo y liderazgo.

Las series de animación utilizadas han sido:
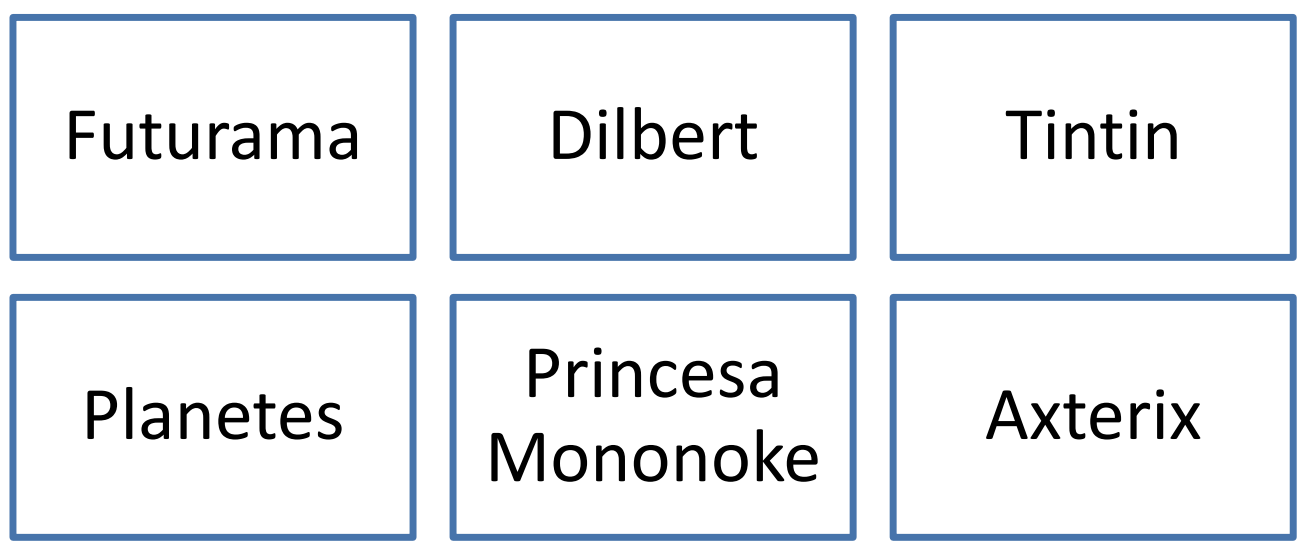

Fig. 1 Algunas series de animación utilizadas en la innovación docente

Los casos prácticos diseñados han sido los siguientes:

(cc) EY-NC-ND 2016, Universitat Politècnica de València 


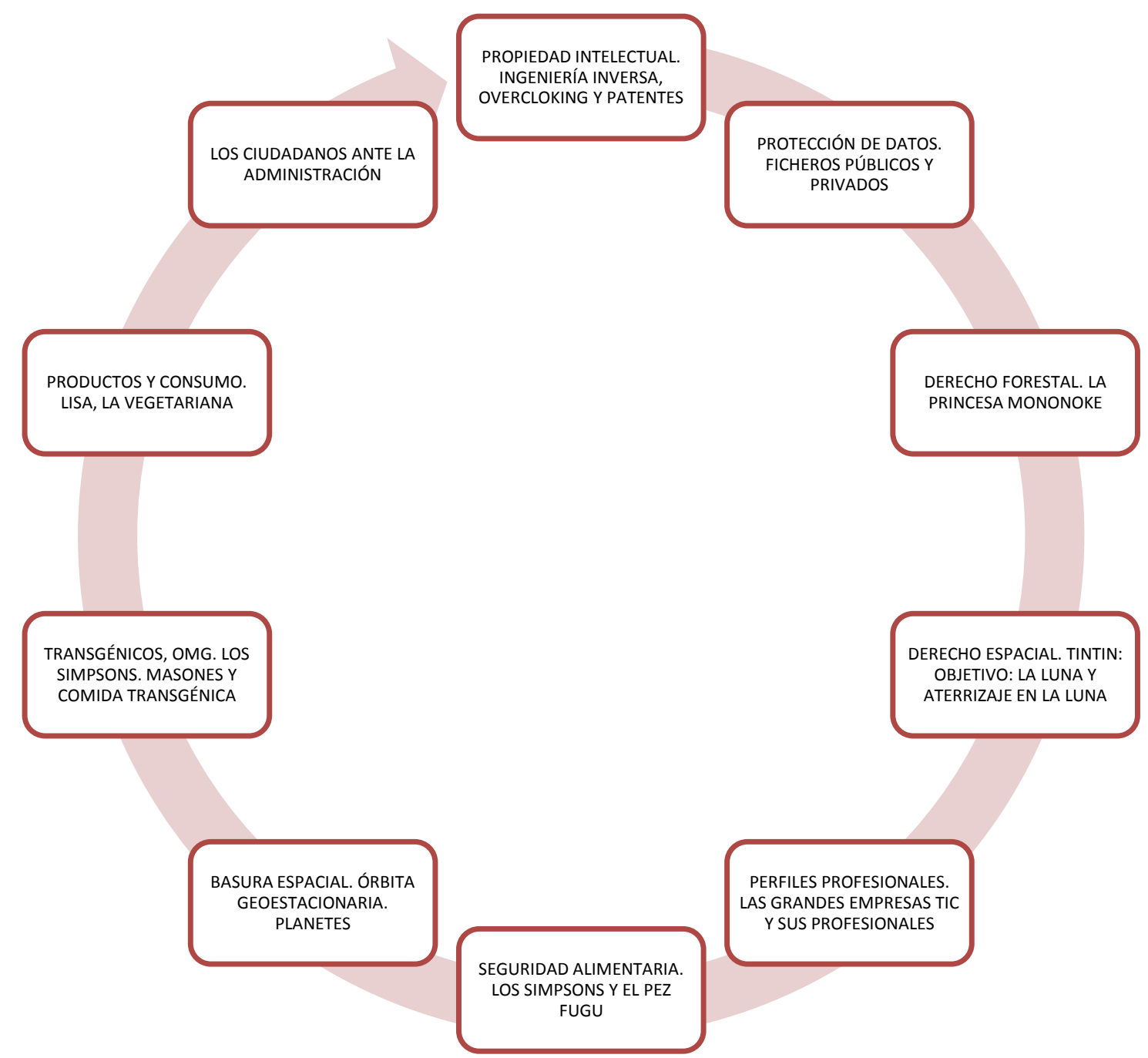

Fig. 2 Algunos casos prácticos diseñados basándose en series de animación (extracto)

Aunque las asignaturas seleccionadas no son punto de control, sí que trabajan la competencia transversal CT-06. Trabajo en Equipo y Liderazgo, en la que, según indica la rúbrica elaborada por el Vicerrectorado de Estudios, Calidad y Acreditación y el Instituto de Ciencias de la Educación y que ha sido trabajada por (ÁBALOS, AGUILAR, ARDID, BELDA, CASTILLA, FERNÁNDEZ, FERRANDO, GUTIÉRREZ, PALOMARES, RAMÓN Y SENDER, 2015) el alumno debe "trabajar y liderar equipos de forma efectiva para la consecución de objetivos comunes de un grupo de personas, contribuyendo al desarrollo personal y profesional de los mismos".

La competencia, según lo indicado por el grupo de trabajo anteriormente referenciado, implica "crear y desarrollar un clima de confianza mutua entre los componentes que permita trabajar de forma responsable y cooperativa". Implica compartir una serie de conocimientos, así como un reparto de tareas y roles. 
Se diferencian los niveles de dominio (primero, segundo y tercero), que obedecen a los dos primeros cursos de grado, los dos últimos cursos de grado y a máster, y se apoya en una serie de indicadores como son la aceptación y cumplimiento de los objetivos, participación en las reuniones; realización de tareas; comunicación y relación, gestión y funcionamiento, seguimiento y control, entre otros.

Consideramos que la implantación de los casos prácticos elaborados con el soporte de las series de animación nos permite trabajar esta competencia, porque se trata de una actividad de trabajo en grupo, en el que se puede observar el grado de cumplimiento de cada uno de los miembros del equipo, así como el desarrollo de los objetivos del equipo (Véase: CANÓS, RAMÓN y SANTANDREU, 2009).

\subsection{Resultados observados en la dinámica de aula con el diseño de casos prácticos basados en series de animación}

Uno de los principales resultados que se observan en el aula es la dinamización del grupo, tras la implantación de un caso práctico basado en series de animación. Los motivos son principalmente que se utilizan las nuevas tecnologías de la información y la comunicación (TICs) y se contextualizan en un diseño de un caso con un recurso didáctico y se pretende que el alumnado pueda detectar los problemas reales extrapolados a un audiovisual, así como aportar distintas soluciones, lo que incrementa la dinámica activa del alumnado dentro del aula.

Hay que tener en cuenta que los casos se utilizan en una tipología de asignatura específica como son las de ciencias sociales y jurídicas que permiten al alumnado formular diversas posiciones, con argumentos contrastables, y que no se trata de asignaturas con una solución inequívoca en el caso planteado.

\subsection{Análisis de los resultados obtenidos en la utilización del caso práctico de la serie Los Simpson en la asignatura de Gestión empresarial de la Edificación}

Durante el curso académico 2015-2016 se ha experimentado la implantación de un episodio de la serie de animación Los Simpson en la asignatura de Gestión empresarial de la Edificación, en el Máster Universitario en Edificación, con la finalidad de observar la respuesta de los alumnos a la utilización de esta innovación docente.

El episodio se refería a la utilización de un blog por parte del protagonista del episodio y la inclusión de información no contrastada, y se integraba dentro de la explicación de los sistemas de información en la empresa de la asignatura.

Para observar la respuesta de los alumnos se diseñaron una serie de preguntas que contestaron de forma anónima.

Las cuestiones que se plantearon fueron las siguientes:

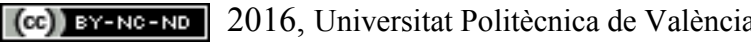

Congreso In-Red (2016) 
a) ¿Qué te ha parecido ver un episodio de animación de forma previa a la resolución de un caso práctico?

b) ¿Has relacionado el episodio de animación con el caso práctico planteado?

c) ¿Qué soluciones aportadas por el episodio de animación consideras que son correctas?

d) ¿Qué soluciones aportadas por el episodio de animación consideras que son incorrectas?

e) Propuestas de mejora de la actividad planteada

Se pasó el cuestionario a un total de 18 alumnos.

Los resultados más destacados que podemos observar en las respuesta son los siguientes:

-La totalidad de los alumnos considera que ha sido una idea muy buena ver el episodio de animación, ya que han entendido mejor el concepto explicado en clase y destacan que ha sido muy acertado realizar un debate final sobre lo visto en el episodio.

Señalan que es una buena idea respecto al aprendizaje y la innovación, ya que se integra el elemento visual, considerando que se aprende mejor con texto enmarcado en un video.

Añaden que el alumnado presta más atención y la clase se hace más amena.

También indican que se dinamiza el contenido de una asignatura, y que resultaría más pesado con el aprendizaje convencional. Y también que se comprenden conceptos que de otra forma resultan muy abstractos.

La crítica que algún alumno hace se centra en que el debate posterior se introducen temas fuera del tema principal, y que el video tendría que ser estrictamente de empresas constructoras. También alguno considera que el episodio tenía duración excesiva.

-Todos los alumnos coinciden en que relacionan el episodio de animación con el caso práctico, ya que se centra en el sistema de información de la empresa.

Inciden que es una forma de profundizar más en el tema, ya que se puede llevar a diversos ámbitos.

Consideran que el episodio de animación es un complemento para resolver el caso práctico de una forma más completa.

-También coinciden totalmente en que las soluciones que aporta el episodio de animación no son viables por estar en contra de la legislación aplicable.

-Destacan en cuanto al uso incorrecto de determinadas situaciones que aparecen en el episodio la utilización inadecuada de las fuentes de información, la información obtenida por medios ilícitos, así como la responsabilidad por daños y perjuicios.

-Respecto a las propuestas de mejora señalan las siguientes:

a) Un episodio que se centre más en el ámbito de la edificación.

b) Ver el episodio por tramos y debate parcial.

c) Resumen previo del episodio.

(cc) EY-NC-ND 2016, Universitat Politècnica de València 
d) Episodio que sea más corto.

e) Mayor tiempo para el debate.

\subsection{Buenas prácticas académicas, propiedad intelectual y deontología en relación con la utilización de casos prácticos basados en series de animación}

Una de las premisas de la utilización de casos prácticos diseñados con series de animación ha sido concienciar al alumnado de varios aspectos:

a) Utilización adecuada de recursos audiovisuales, tanto en el visionado de los casos prácticos, como en la utilización de los mismos para la elaboración de las prácticas.

b) Concienciación de la propiedad intelectual de la obra audivisual, en el sentido de la utilización del derecho de cita y la evitación de reproducción de contenidos ajenos sin utilizar dicho derecho (RAMÓN, 2013).

c) Fomento de las buenas prácticas académicas, siguiendo las directrices que ya ha marcado la Escuela Técnica Superior de Ingenieros Industriales de la Universitat Politècnica de València, referente a la normativa de honestidad académica, aprobada en Junta de Escuela de 15 de julio de 2014.

El Real Decreto 1791/2010, de 30 de diciembre, por el que se aprueba el Estatuto del Estudiante Universitario indica, en su artículo 13, que: "los estudiantes deben abstenerse de la utilización o cooperación en procedimientos fraudulentos en las pruebas de evaluación, en los trabajos que se realicen, o en documentos oficiales de la universidad".

También señalar el Decreto de 8 de septiembre de 1954 por el que se aprueba el Reglamento de disciplina académica de los Centros Oficiales de Enseñanza Superior y de Enseñanza Técnica dependientes del Ministerio de Educación Nacional, aunque está parcialmente derogado.

Por su parte, la política de integridad científica y buenas prácticas en investigación en la Universitat Politècnica de València, establece que se considera mala práctica científica, en el punto I: "la fabricación, falsificación o plagio en la propuesta, realización o revisión de investigaciones o en la publicación de sus resultados. También la alteración de los fines de la investigación y la manipulación de la voluntad de otras personas, objetos de investigación y/o colaboradores, son atentados graves contra la integridad científica y la buena praxis en investigación".

\section{Conclusiones}

Hemos considerado que las nuevas tecnologías de la información son elementos que facilitan los métodos activos en la transmisión de competencias (RAMÓN y SAZ, 2004), y que la utilización de recursos audiovisuales nos permiten dinamizar la actividad docente en el aula (RAMÓN, GIMÉNEZ, CABEDO, CASAR Y OLTRA, 2012; RAMÓN, CASAR y GIMÉNEZ, 2014).

(c)) EY-NC-ND 2016, Universitat Politècnica de València

Congreso In-Red (2016) 
Los casos prácticos elaborados tomando como base series de animación nos ha permitido dinamizar las asignaturas, así como una mayor involucración del alumnado, y un fomento de la reflexión sobre las cuestiones planteadas.

La contextualización en situaciones descritas en las series de animación elegidas también han permitido al alumnado aplicar la legislación para aportar soluciones a los conflictos, teniendo en cuenta la multidisciplinariedad de las asignaturas.

La experiencia y resultados obtenidos en la utilización de un episodio concreto de una serie de animación en una asignatura durante el presente curso académico nos ha permitido obtener la impresión del alumnado, a través de la contestación al cuestionario indicado de su excelente valoración de la utilización de series de animación como contextualización de casos prácticos. Resulta muy interesante las propuestas de mejora del alumno, que se tendrán en cuenta para futuros casos.

En el caso concreto de la aplicación en la asignatura de Gestión empresarial de la Edificación, en el que se ha aplicado la dinámica de casos basados en series de animación, a un total de 23 alumnos, la respuesta ha sido muy positiva tanto por parte del discente como también se resalta la mayor participación en el aula, lo que ha aumentado la interactividad por parte del alumnado y la aportación crítica a situaciones ficticias, pero con una aplicación real.

\section{Agradecimientos}

Trabajo realizado en el marco del PIME «Utilización de las series de animación como recurso didáctico en la elaboración de casos» de la Universitat Politècnica de València, 2015-2016; Proyecto MINECO (DER2012-37844) siendo el Investigador Principal el Dr. D. Lorenzo Cotino Hueso, Catedrático acreditado de Derecho constitucional, Universitat de València-Estudi General, del Proyecto MINECO (DER2013-4256R), siendo los Investigadores Principales la Dra. $D^{\mathrm{a}}$. Luz María Martínez Velencoso, Profesora Titular de Derecho civil, Universitat de València-Estudi General, y el Dr. D. Javier Plaza Penadés, Catedrático de Derecho civil, Universitat de València-Estudi General, y Proyecto «Derecho civil valenciano y europeo» del Programa Prometeo para Grupos de Investigación de Excelencia de la Conselleria de Educación, Cultura y Deporte, GVPROMETEOII2015-014 y del Microcluster «Estudios de Derecho y empresa sobre TICs (Law and business studies on ICT)», dentro del VLC/Campus, Campus de Excelencia Internacional (International Campus of Excellence), coordinado por el Dr. D. Javier Plaza Penadés, Catedrático de Derecho civil, Universitat de València-Estudi General, y Proyecto I+D Excelencia del Ministerio y Competitividad. "El avance del Gobierno Abierto. Régimen jurídico constitucional de la implantación de políticas de transparencia, acceso a la información, datos abiertos, colaboración y participación especialmente a través de las TIC y del Gobierno electrónico". DER2015-65810-P (2016-2018). Investigador Principal: Dr. D. Lorenzo Cotino Hueso. Catedrático acreditado de Derecho constitucional. Universitat de València 


\section{Referencias}

Ábalos Galcerá, A., Aguilar Botija, A., Ardid Ramírez, M., Belda Navarro, R.Ma., Castilla Cabanes, N., Fernández Méndez, Ma. M., Ferrando Bataller, M., Gutiérrez Colomer, R. P., Palomares Chust, A., ramón fernández, F. y Sender Contell, M. (2015): Rúbrica CT-06. Trabajo en equipo y liderazgo. Valencia: Instituto Ciencias de la Educación. Universitat Politècnica de València.

ANTINORA, S. (2010). "The Simpsons, Gender Roles, and Witchcraft: the witch in modern popular culture". $452^{\circ} \mathrm{F}$ : revista de teoría de la literatura y literatura comparada, núm. 3, págs. 115-131.

$<$ http://www.452f.com/images/pdf/numero03/antinora/03 452f mono antinora trad es.pdf $>$

[Consulta: 20 de enero de 2016].

CANÓS DARÓS, L. y RAMÓN FERNÁNDEZ, F. (2005): "Aplicación de las nuevas tecnologías para el trabajo autónomo del alumno". Actas del XX Simposium Nacional de la Unión Científica Internacional de Radio. URSI 2005, Gandía: Universitat Politècnica de València, págs. 1-4.

CANÓS DARÓS, L. y RAMÓN FERNÁNDEZ, F. (2007): "La aplicación de las TICs en el aprendizaje de disciplinas jurídicas aplicadas en carreras técnicas en el marco de la convergencia europea". Conocimiento Abierto, Sociedad Libre, Actas digitales del III Congreso ONLINE del Observatorio para la CiberSociedad, Barcelona: Observatorio para la CiberSociedad, págs. 1-7.

CANÓS DARÓS, L. y RAMÓN FERNÁNDEZ, F. (2009): "La calidad de la docencia respecto a las nuevas tecnologías y recursos didácticos". Autogestión, cooperación y participación en las Ciencias Sociales, Valencia, págs. 341-352.

CANÓS DARÓs, L. y RAmón fernández, F. y Albaladejo Fernández-Silgado, M. (2008): "Los roles docentes y discentes ante las nuevas tecnologías y el proceso de convergencia europea". $C D$ Resúmenes comunicaciones $V$ Congreso Iberoamericano de Docencia Universitaria, Valencia: AIDU. Asociación Iberoamericana de Didáctica Universitaria, Universidad Politécnica de Valencia e Instituto Ciencias de la Educación, 388-390.

CANÓS Darós, L. y RAmón fernández, F. y Albaladejo Fernández-Silgado, M. (2009): "Los roles docentes y discentes ante las nuevas tecnologías y el proceso de convergencia europea". Actas $V$ Congreso Iberoamericano de Docencia Universitaria. Valencia: Universidad Politécnica de Valencia.

CANÓS Darós, L. y RAMÓn Fernández, F. y SANTANDReu Mascarell, C. (2009): "Ventajas y desventajas del uso de las nuevas tecnologías en la adquisición de competencias profesionales". Crisis analógica, futuro digital. Actas IV Congreso ONLINE de la Cibersociedad 2009.

ESCUELA TECNICA SUPERIOR DE INGENIEROS INDUSTRIALES DE LA UNIVERSITAT POLITECNICA DE VALENCIA (2014). Normativa de honestidad académica.

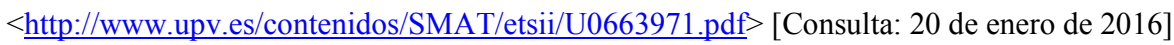

España Decreto de 8 de septiembre de 1954 por el que se aprueba el Reglamento de disciplina académica de los Centros oficiales de Enseñanza Superior y de Enseñanza Técnica dependientes del Ministerio de Educación Nacional. BOE, 12 de octubre de 1954, núm. 285, p. 6863-6866..

España. Real Decreto 1791/2010, de 30 de diciembre, por el que se aprueba el Estatuto del Estudiante Universitario. BOE, 31 de diciembre de 2010, núm. 318, p. 109353-109380.

ÍNIIGO JURADO, A.I. (2012): "Las series de animación infantiles. Un producto global que pretende ser educativo". Ficcionando: series de televisión a la española, Belén Puebla Martínez, Elena Carrillo y Ana Isabel Íñigo Jurado (eds.), Madrid: Fragua, págs. 95-117.

(c)) EY-NC-ND 2016, Universitat Politècnica de València

Congreso In-Red (2016) 
Lazo, C. M. y tovar laheras, A. (2011): "Los Simpson, un fenómeno social con 20 años de permanencia en la programación televisiva”. Revista Mediterránea de Comunicación, vol. 2, págs. 143-157.

MARTÍNEZ SIERRA, J.J. (2004): Estudio descriptivo y discursivo de la traducción del humor en textos audiovisuales. El caso de los Simpson, tesis doctoral dirigida por Frederic Chaume Varela. Castellón: Universitat Jaume I.

$<\underline{\text { http://www.tdx.cat/handle/10803/10566 }}>$ [Consulta: 20 de enero de 2016].

POLÍTICA DE INTEGRIDAD CIENTÍFICA Y BUENAS PRÁCTICAS EN INVESTIGACIÓN EN LA UNIVERSITAT POLITĖCNICA DE VALÈNCIA

$<$ http://poliscience.blogs.upv.es/files/2012/09/Politicas-de-integridad-cientifica-y-buenaspracticas.pdf $>$ [Consulta: 23 de enero de 2016].

PORTILLO FERNÁNDEZ, J. (2015): La interpretación inferencial en la comunicación absurda: (Aplicado a un programa de Matt Groening). Tesis doctoral dirigida por Catalina Fuentes Rodríguez y Francisco J. Salguero Lamillar. Sevilla: Universidad de Sevilla.

$<$ http://fondosdigitales.us.es/tesis/tesis/2614/la-interpretacion-inferencial-en-la-comunicacionabsurda-aplicado-un-programa-de-matt-groening/> [Consulta: 20 de enero de 2016].

RAMÓN FERNÁNDEZ, F. (2013): "La redefinición de las excepciones en materia de propiedad intelectual. Derecho de cita, plagio e internet". Libertad de expresión e información en internet. Amenazas y protección de los derechos personales, Coordinador Lorenzo Cotino Hueso y Loreto Corredoira Alfonso. Madrid: Centro de Estudios Políticos y Constitucionales, págs. 233-258.

RAMÓN FERNÁNDEZ, F. y SAZ GIL, Ma. I. (2004): “Las nuevas tecnologías como elemento facilitador de los métodos activos en la transmisión de competencias". Hacia una docencia de calidad: políticas y experiencias. Actas del I Congreso de la Red Estatal de Docencia Universitaria y III Jornada de Mejora Educativa de la Universitat Jaume I. Castellón: Publicaciones Universitat Jaume I de Castelló, págs. 330-341.

RAMÓN FERNÁNDEZ,F., CASAR FURIÓ, M. E. y GIMÉNEZ CHORNET, V. (2014): "La aplicación de las TICs a través de la utilización de bases de datos y recursos online en la docencia". Jornadas de innovación educativa y docencia en red (IN-RED 2014), Valencia: Universitat Politècnica de València, págs. 130-139.

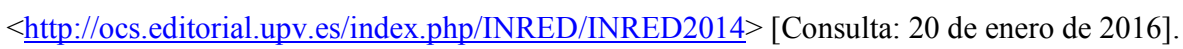

RAMÓN FERNÁNDEZ, F., GIMÉNEZ CHORNET, V., CABEDO MALlOL, V., CASAR FURIÓ, M. E. y OLTRA GUTIÉRREZ, J. V. (2012): "La comunicación 2.0". V Jornada de Innovación Docente en la Escuela Técnica Superior de Ingeniería Informática, JIDINF 2012, Valencia.

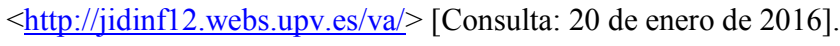

ROMERo CARMONA, V. (2006): "Buscando a Homer. La influencia de las series de animación en la comedia gamberra”. Secuencias: revista de historia del cine, núm. 24, págs. 4363.

$<$ https://repositorio.uam.es/xmlui/bitstream/handle/10486/3925/27367_24.4.pdf?sequence=1>

[Consulta: 20 de enero de 2016].

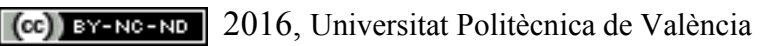

Congreso IN-RED (2016) 